\title{
Publisher's Note: Josephson junction detectors for Majorana modes and Dirac fermions
} [Phys. Rev. B 92, 224501 (2015)]

\author{
M. Maiti, K. V. Kulikov, K. Sengupta, and Yu. M. Shukrinov
}

Q (Received 15 June 2018; published 21 June 2018)

DOI: 10.1103/PhysRevB.97.219904

This paper was published online on 1 December 2015 with an error in the author list. The name of the second author should read as "K.V. Kulikov." The author name has been corrected as of 18 June 2018. The author name is incorrect in the printed version of the journal. 